\title{
The changing situation about maternal age, risk factors and pregnancy outcomes after the two-child policy: a retrospective cohort study
}

\author{
Xiaohui Teng ${ }^{1,2}$, Mc Intyre Shane ${ }^{2}$, Shilei $\operatorname{Pan}^{2}$ \\ ${ }^{1}$ Department of Health Management Center, Xiang'an Hospital of Xiamen University of Fujian Province, Xiamen 361101, China; ${ }^{2}$ Department \\ of Gynaecology and Obstetrics, Zhujiang Hospital of Southern Medical University, The Second School of Clinical Medicine, Southern Medical \\ University, Guangzhou 510515, China \\ Contributions: (I) Conception and design: X Teng, S Pan; (II) Administrative support: X Teng, MI Shane; (III) Provision of study materials or patients: \\ All authors; (IV) Collection and assembly of data: X Teng, MI Shane; (V) Data analysis and interpretation: All authors; (VI) Manuscript writing: All \\ authors; (VII) Final approval of manuscript: All authors. \\ Correspondence to: Shilei Pan. Department of Gynaecology and Obstetrics, Zhujiang Hospital of Southern Medical University, The Second School of \\ Clinical Medicine, Southern Medical University, Guangzhou 510515, China. Email: semysci@sina.com.
}

Background: To evaluate the relationship between gestational age, risk factors, pregnancy outcomes, and the causes and trends of the changes in the incidence of pregnancy in different years after the two-child policy.

Methods: The study enrolled all of the childbirth women $(n=7,016)$ from January 1st, 2015 to December 31th, 2017 in the Zhujiang Hospital. According to the age of all childbirth women, they were divided into four groups: $\leq 24,25-29,30-34$ and $\geq 35$ years old. While 20-29 years old group regarded as the control group $(\mathrm{OR}=1)$. Pregnancy complications and outcomes data of 4 groups were collected. $\chi^{2}$ test and binarylogistic regression used as the analyze method. Stratified chi-square used to compare the rates between the pregnancy risk factors, childbirth modes and pregnancy outcomes in different years, which may be affected by the age.

Results: Compared with the other groups, women in $\geq 35$ years old showed the highest incidences of chronic medical complications and pregnancy induced complications, so as the abnormal neonatal birth outcomes. While women in $\leq 24$ years old displayed a high risk of preeclampsia. After the childbearing policy change, the maternal age ratio increased year by year. The incidences of various risk factors, delivery modes and adverse pregnancy outcomes were also changed between different years and different age groups. The differences of annual incidence showed a statistical significance besides oligohydramnios.

Conclusions: The study indicated that the changes in childbearing policy lead to the changes of age distribution proportion, which finally caused the changes of risk factors incidence. Furthermore, the advance of medical technology and society also affected the changes of pregnancy risk factors, delivery modes and the pregnancy outcomes in different years.

Keywords: Two-child policy; age; high risk factor; chronic medical complications; pregnancy induced complications

Submitted Sep 30, 2019. Accepted for publication Feb 12, 2020.

doi: $10.21037 /$ apm.2020.04.27

View this article at: http://dx.doi.org/10.21037/apm.2020.04.27 


\section{Introduction}

Fertility intention is the advanced variable affecting the actual fertility level, providing important information for predicting fertility level (1). The implementation of family planning policy (FPP) in whole of China has been started in the early 1970's. It is a forced choice under the longterm downturn economic development and population explosion (2). The FPP has been doing dynamic adjustment based on changes of the population, economic and social situation. In China, since the end of 2013, the two-child policy has been implemented throughout the country (3). While with the economic and social development and the change of the population situation, the policy direction of the universal two children is bound to develop (4). Furthermore, the eighteenth meeting of the twelfth NPC Standing Committee has adopted the policy of carrying out the comprehensive two-child policy from January 1st, 2016 (3-5). At present, the universal two-child policy is being implemented in all parts of China.

The universal two-child policy is a continuation of FPP adjustment. It marks the end of the tightening FPP and the beginning of a relatively liberal of FPP. Its demographic effects are expected to benefit far outweigh the disadvantages $(6,7)$. While China had introduced one-child policy for more than 20 years, two-child policy and the universal two-child policy are gradually being enforced in recent years $(8,9)$. The changed policy will cause the couple who are eager to have a second child for several years even more than 10 years, to be able to conceive in a short period of time. While due to the worry about the growing age that may influence the fertility, in recent years, the number of advanced pregnant women who are eligible for the new policy is higher and is increasing year by year $(1,9-11)$.

In addition, due to the changes of age distribution proportion for the fertility, the incidence of the pregnancy risk factors, delivery modes and the pregnancy outcomes will be more common and continue increasing in different ages and different couples. Thus, in this study, we enrolled 7,016 childbirth women from January 1st 2015 to December 31th 2017 in the Zhujiang Hospital as the research object to analyze the relationship between gestational age, risk factors, pregnancy outcomes, and the change causes and trend of that incidence in different years after the two-child policy. Analyze whether the incidence of change among different years have statistical significance, and explore the situation of the changing incidence. And then discuss the situation of the risk factors, guide the future of health care and treatment in the perinatal period, improve the highrisk pregnancy supervision and management, to ensure the maternal and child health.

\section{Methods}

\section{Research object}

Take 7,016 childbirth women from January 1st 2015 to December 31th 2017 in the Zhujiang Hospital as the research object. The age of all childbirth women were 15-48 (average age $29.73 \pm 4.80$ ) years old, and they all had reached 28 weeks of gestational age.

\section{Study design}

Since the number of $\leq 20$ and $\geq 40$ years old of childbirth women were less (only 123 cases and 200 cases respectively), so we divided the cases into four groups according to age: $\leq 24$ years old (860 cases), 25-29 years old (2,792 cases), $30-34$ years old (2,214 cases), $\geq 35$ years old (1,150 cases). And we also divided the cases into three groups according to the years and delivery dates: with the year 2015 (the year after opening unilateral two-child policy that one of the couple is one-child) contained 2,078 cases, the year 2016 (the year of the early stage of the open comprehensive twochild policy that ignore whatever the couple is one-child or not) contained 2,371 cases, and the year 2017 (the year after opening the comprehensive two-child policy) contained 2,567 cases.

In addition, according to the cases in the four groups of $\leq 24$ years old (860 cases, $12.3 \%), 25-29$ years old $(2,792$ cases, $39.8 \%), 30-34$ years old (2,214 cases, $31.6 \%)$, $\geq 35$ years old ( 1,150 cases, $16.4 \%$ ), the age number of 25 to 29 years old accounted for the most, which is also the best reproductive age for women. This showed a normal reproductive age distribution. Thus, according this, we put the age of 20-29 years old as the control group, and the rest of the age groups were the research teams.

The study was approved by ethics committee of The IRB of Zhujiang Hospital of Southern Medical University (No. 2018-FCK-001), and all the participants have gave informed consent before taking part.

\section{Analyze data and method}

The main chronic medical complications of pregnant women among all age groups included heart disease, anemia, uterine 
Table 1 Medical history of adverse pregnancy \& IVF-ET postoperative in different age (\%)

\begin{tabular}{lcccc}
\hline Groups & $\leq 24$ Y/O & $25-29$ Y/O & $30-34$ Y/O & $\geq 35$ Y/O \\
\hline Medical history of adverse pregnancy & $18(2.09)$ & $93(3.33)$ & $127(5.74)$ & $89(7.74)$ \\
IVF-ET postoperative & $3(0.35)$ & $25(0.90)$ & $68(3.07)$ & $27(2.35)$ \\
\hline
\end{tabular}

IVF-ET, in vitro fertilization and embryo transplantation.

Table 2 Binary-Logistic regression for the histories of abnormal pregnancy \& IVF-ET in different age

\begin{tabular}{lccr}
\hline \multirow{2}{*}{ Group } & \multicolumn{3}{c}{$\mathrm{P}, \mathrm{OR}(95 \% \mathrm{Cl})$} \\
\cline { 2 - 4 } & $\mathrm{S} 24 \mathrm{Y} / \mathrm{O}(\mathrm{n}=860)$ & $30-34 \mathrm{Y} / \mathrm{O}(\mathrm{n}=2,214)$ & $\geq 35 \mathrm{Y} / \mathrm{O}(\mathrm{n}=1,150)$ \\
\hline Medical history of adverse pregnancy & $0.06,-$ & $0.00,1.77(1.34-2.32)$ & $0.00,2.43(1.81-3.28)$ \\
IVF-ET postoperative & $0.12,-$ & $0.00,3.51(2.21-5.57)$ & $0.00,4.72(2.89-7.70)$ \\
\hline
\end{tabular}

IVF-ET, in vitro fertilization and embryo transplantation.

leiomyoma, diabetes mellitus (DM), chronic hypertension and abnormal thyroid function. Pregnancy induced complications contained gestational diabetes mellitus (GDM), premature rupture of membranes (PROM), hydramnios, oligohydramnios, placenta previa, placental abruption, scar uterus, multiple pregnancy, preeclampsia and postpartum hemorrhage. Delivery modes involved vaginal delivery and cesarean delivery. Common adverse pregnancy outcomes contained full-term underweight infant, fetal macrosomia (neonatal weight $\geq 4,000 \mathrm{~g}$ ), low birth weight infant (neonatal weight $<2,500 \mathrm{~g}$ ), premature infant, birth defects and perinatal-death infant.

We also collected and analyzed the cases of medical history of adverse pregnancy and the pregnancy which were assisted reproductive technology (ART) by in vitro fertilization and embryo transplantation (IVF-ET). The medical history of adverse pregnancy was referred to the previous history of spontaneous abortion, embryo damage, and fetal deformities of maternal.

\section{Statistical method}

All the data were displayed as mean \pm standard deviation $(\bar{x}$ $\pm s)$ and percentage. The analyze method of chi-square test were used as compared the data between multiple groups, the binary Logistic regression analysis were applied to pregnancy associated risk factors analysis, and the layered chi-square analyze method was used to compare annual incidences of various risk factors and the birth outcomes through the statistical software SPSS 22.0. The OR values of the control group (25-29 years old) was set as 1 . $\mathrm{P}<0.05$ for the difference regard as statistically significant.

\section{Results}

\section{The situation analysis for medical history of adverse pregnancy and assisted reproduction in pregnant pregnancy of different age}

The medical history of adverse pregnancy in different age and the assisted reproductive help pregnancy for "testtube baby" like "IVF-ET" postoperative pregnancies is shown in Table 1. Binary Logistic regression analysis of the medical history of adverse pregnancy (327 cases), the incidence of pregnancy with assisted reproductive method (143 cases) and the control group (OR =1) showed in Table 2 . The results of the medical history of adverse pregnancy and assisted pregnancy showed that the ORs values were $>1$ at the group of 30-34 and 35 years old, which indicated that pregnant women after the age of 30 have a risk factor for the medical history of adverse pregnancy and auxiliary reproductive assisted pregnancy, and it also indicated that with the increase of age, the increase risk factor for pregnancy.

\section{The correlation analysis of the chronic medical complications of pregnancy and the pregnancy induced complications in different ages}

In this study, the chronic medical complications of pregnancy were: heart disease (41 cases), anemia (631 cases), uterine leiomyoma (154 cases), diabetes ( 35 cases), chronic hypertension (30 cases) and 303 cases of thyroid dysfunction, 
Table 3 The chronic medical complications of pregnancy and the pregnancy induced complications in different ages (\%)

\begin{tabular}{|c|c|c|c|c|}
\hline Group & $\leq 24 \mathrm{Y} / \mathrm{O}$ & $25-29 \mathrm{Y} / \mathrm{O}$ & $30-34 \mathrm{Y} / \mathrm{O}$ & $\geq 35 \mathrm{Y} / \mathrm{O}$ \\
\hline Anemia & $74(8.60)$ & $264(9.46)$ & $192(8.67)$ & $101(8.78)$ \\
\hline Uterine leiomyoma & $3(0.35)$ & $26(0.93)$ & $62(2.80)$ & $63(5.48)$ \\
\hline DM & $1(0.12)$ & $7(0.25)$ & $10(0.45)$ & $17(1.48)$ \\
\hline Thyroid dysfunction & $27(3.14)$ & $106(3.80)$ & $110(4.97)$ & $60(5.22)$ \\
\hline GDM & $97(11.28)$ & $546(19.56)$ & $585(26.42)$ & $427(37.13)$ \\
\hline PROM & $144(16.74)$ & $494(17.69)$ & $370(16.71)$ & $186(16.17)$ \\
\hline Hydramnios & $12(1.40)$ & $39(1.40)$ & $36(1.63)$ & $34(2.96)$ \\
\hline Placental abruption & $4(0.47)$ & $19(0.68)$ & $18(0.81)$ & $10(0.87)$ \\
\hline Scar uterus & $36(4.19)$ & $288(10.32)$ & $603(27.24)$ & $449(39.04)$ \\
\hline Multiple pregnancies & $9(1.05)$ & $38(1.36)$ & $46(2.08)$ & $34(2.96)$ \\
\hline Preeclampsia & $19(2.21)$ & $30(1.07)$ & $33(1.49)$ & $35(3.04)$ \\
\hline Postpartum hemorrhage & $15(1.74)$ & $33(1.18)$ & $34(1.54)$ & $30(2.61)$ \\
\hline
\end{tabular}

DM, diabetes mellitus; GDM, gestational diabetes mellitus; PROM, premature rupture of membranes.

including 123 cases of hyperthyroidism and 180 cases of hypothyroidism. Pregnancy induced complications were: GDM (1,655 cases), PROM (1,194 cases), hydramnios (121 cases), oligohydramnios (226 cases), placenta previa (72 cases), placental abruption (51 cases), scar uterus (1,376 cases), multiple pregnancy (127 cases), preeclampsia (117 cases), postpartum hemorrhage (112 cases). Different age groups of chronic medical complications of pregnancy and the pregnancy induced complications are shown in Table 3. The binary logistic regression analysis was used to analyze the incidence of pregnancy complications in all age groups in Table 3. The OR of the control group was set as 1 . While Table 4 showed the correlation analysis of the chronic medical complications of pregnancy and the pregnancy induced complications in different ages.

From Table 4, we could see that in the age group of $30-34,35$ years old, the OR values were $>1$, which indicated that pregnancy with uterine leiomyoma and abnormal thyroid function will become the high risk factor after age 30 for pregnancy women, and with the increase of age, the risk factors were increased. The OR $>1$ in 35 age group for DM and chronic hypertension. Moreover, in the age of $\leq 24$ years old, the ORs were $<1$ for GDM and scar uterus, and in $30-34$ and $\geq 35$ years old, the ORs were $>1$, that shows with the age increased, the OR values increased. It suggested that childbearing age in $\leq 24$ years old group was the protection factor of GDM and scar uterus; while in the age of 30 or higher was their risk factor.

In addition, placenta previa, multiple pregnancy and postpartum hemorrhage, in the age group of $\geq 35$ years old, the ORs were $>1$, which explained that in the advanced maternal age ( $\geq 35$ years), age was their risk factor. Preeclampsia in age $\leq 24$ years old group and $\geq 35$ years old group ORs were $>1$, It meant that in those two age groups, age was the risk factor. Oligohydramnios in 30 34 years old group, the OR value $<1$, that meant in this group, age was the protect factor of oligohydramnios. While in $\geq 35$ years old group, hydramnios' OR was $>1$, described that the advanced maternal age was the high-risk factor of hydramnios.

\section{The delivery modes and the adverse pregnancy outcomes in different age situation analysis}

In this study, there were 4,789 cases of vaginal delivery $(68.3 \%)$ and 2,227 cases of cesarean delivery (31.7\%). 
Table 4 Binary-Logistic regression for chronic medical complications of pregnant and the pregnancy induced complications in different ages

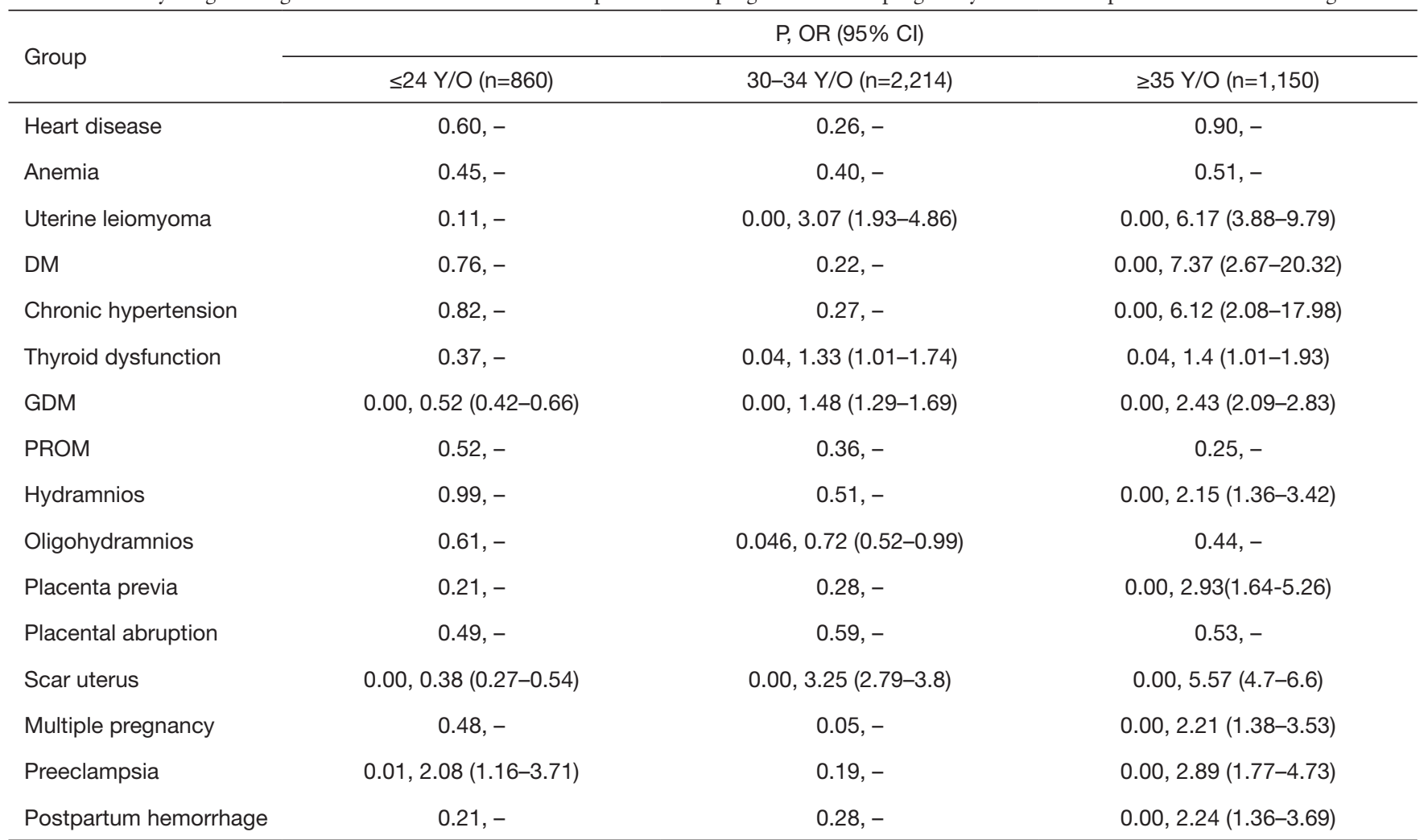

DM, diabetes mellitus; GDM, gestational diabetes mellitus; PROM, premature rupture of membranes.

Table 5 Delivery modes and adverse pregnancy outcomes in different ages (\%)

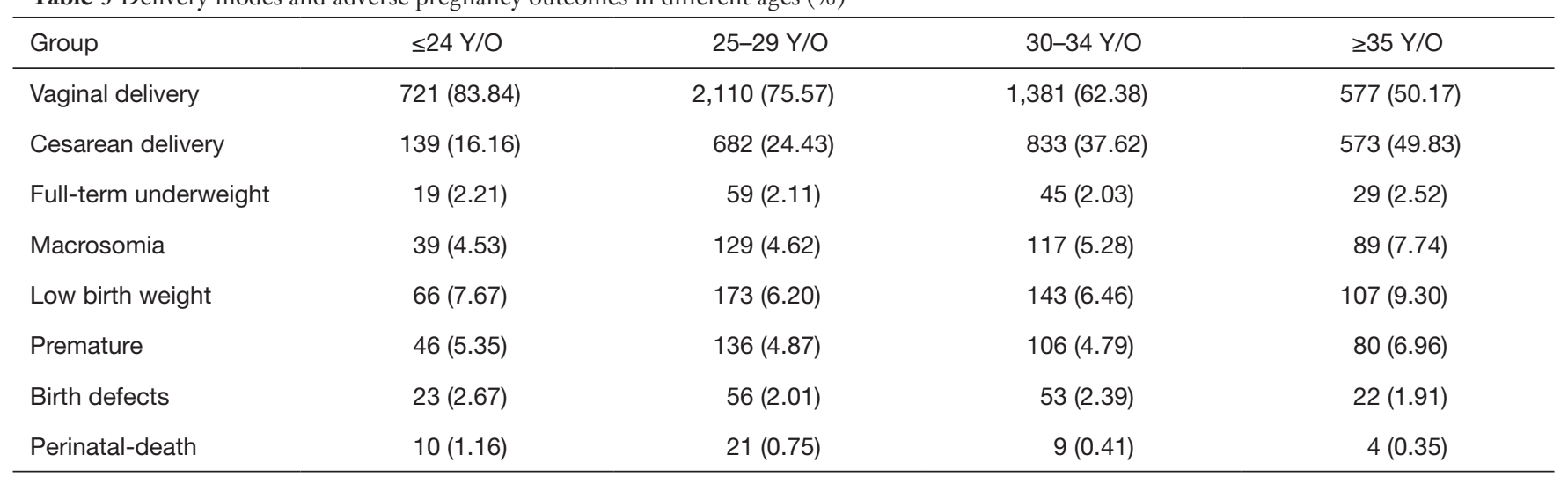

There were also 152 cases of full-term underweight infants, 374 cases of fetal macrosomia, 489 cases of low birth weight infants, 368 cases of premature infants, 154 cases of birth defects and 44 cases of perinatal-death infants. Each delivery modes and the adverse pregnancy outcomes are shown in Table 5. Data and the binary Logistic regression analysis, whose control group is $25-29$ age group $(n=2,792$,
OR =1), are shown in Table 6 .

The results showed that with the age increased, OR of vaginal delivery reduced gradually, the OR of cesarean delivery increased, and the difference was statistically significant. Macrosomia in $\leq 24$ years old group OR was $<1$, while in $\geq 35$ years old age group $\mathrm{OR}$ was $>1$, which indicated childbearing age in $\leq 24$ years old group was the 
Table 6 Binary-Logistic regression for delivery modes and adverse pregnancy outcomes in different ages

\begin{tabular}{|c|c|c|c|}
\hline Group & \multicolumn{3}{|c|}{ P, OR (95\% Cl) } \\
\hline Vaginal delivery & $0.00,1.68(1.37-2.05)$ & $0.00,0.54(0.47-0.61)$ & $0.00,0.33(0.29-0.38)$ \\
\hline Cesarean delivery & $0.00,0.6(0.49-0.73)$ & $0.00,1.87(1.66-2.11)$ & $0.00,3.07(2.67-3.55)$ \\
\hline Full-term underweight & $0.87,-$ & $0.84,-$ & $0.43,-$ \\
\hline Low birth weight & $0.13,-$ & $0.70,-$ & $0.00,1.56(1.21-2.0)$ \\
\hline Premature & $0.57,-$ & $0.89,-$ & $0.01,1.46(1.1-1.94)$ \\
\hline Birth defects & $0.24,-$ & $0.35,-$ & $0.85,-$ \\
\hline Perinatal-death & $0.26,-$ & $0.12,-$ & $0.16,-$ \\
\hline
\end{tabular}

protection factor for macrosomia but in the age of 35 or higher was its risk factor. In $\geq 35$ years old group of low birth weight infants and premature infants OR was $>1$, that implicated the advanced maternal age was the risk factor of them.

\section{The incidence of change between different years statistical significance, and the change trend of incidence}

Analysis of the risk factors for pregnancy and birth outcomes in previous research whose results of the ORs were $>$ or $<1$ and $\mathrm{P}$ values were significant. Statistics about the incidences of various risk factors and the delivery modes according to different age groups and different years is illustrated in Table 7. The result showed that besides the oligohydramnios, the high-risk factors and incidence of contrast $\mathrm{P}<0.05$, which suggested that in addition to oligohydramnios, the difference of annual incidence had statistical significance.

\section{Discussion}

From the combined data over the 3 years, maternal age accounted for the most significant increase, from $12.6 \%$ in 2015 to $20.4 \%$ in 2017 . Wei et al. (12) once researched 110,450 cases in 13 different provinces in China in 2011 (including Guangdong province), about pregnancy risk factors statistical samples, and found that older maternal women accounted for $10.02 \%(11,072 / 110,450)$. As the two-child policy has been carried out continuously, the proportion of advanced maternal women increased year by year, and in different years, the incidences of various risk factors occurring at different ages were also different (13).

In our study, from the statistical results of chronic medical complications of pregnancy, it could be concluded that the incidence of the pregnant women after age 30 had significant increased in medical history of adverse pregnancy, pregnancy by ART, uterine leiomyoma and thyroid dysfunction. The incidences of chronic hypertension and DM in advanced maternal age increased obviously, which also consistent with the previous researches $(14,15)$. Furthermore, with the development of our social economy and childbearing policy adjustment, advanced maternal women presented with a sharp rise in recent times. Because of the advanced age, female fertility also declined, the women who had a strong desire to conceive had to resort to ART. So, the medical history of adverse pregnancy and pregnancy by ART incidence increased year by year.

By the statistical results in the study, we found that maternal presence of medical history of adverse pregnancy, whose incidence was $4.23 \%$ in 2015 , slightly decreased to $3.29 \%$ in 2016 , then significantly increased to $6.27 \%$ in 2017. The reason may because in the early start period of fully open "two-child policy" (the year in 2016), the women tried to get pregnant, but due to the adverse maternal age, had limitations in successfully becoming pregnant. They needed time to prepare and necessary treatment for any form of primary diseases, and only then afterwards could plan for a successful pregnancy. So, in 2017 the incidence increased obviously more than before. On the other hand, the result show that the pregnancy incidence by ART in 2015 was $2.07 \%$, and then slightly decreased to $1.86 \%$ in 2016 , while it rebounded to $2.18 \%$ in 2017 , basically mirroring the incidence in 2015 . The reason for that 


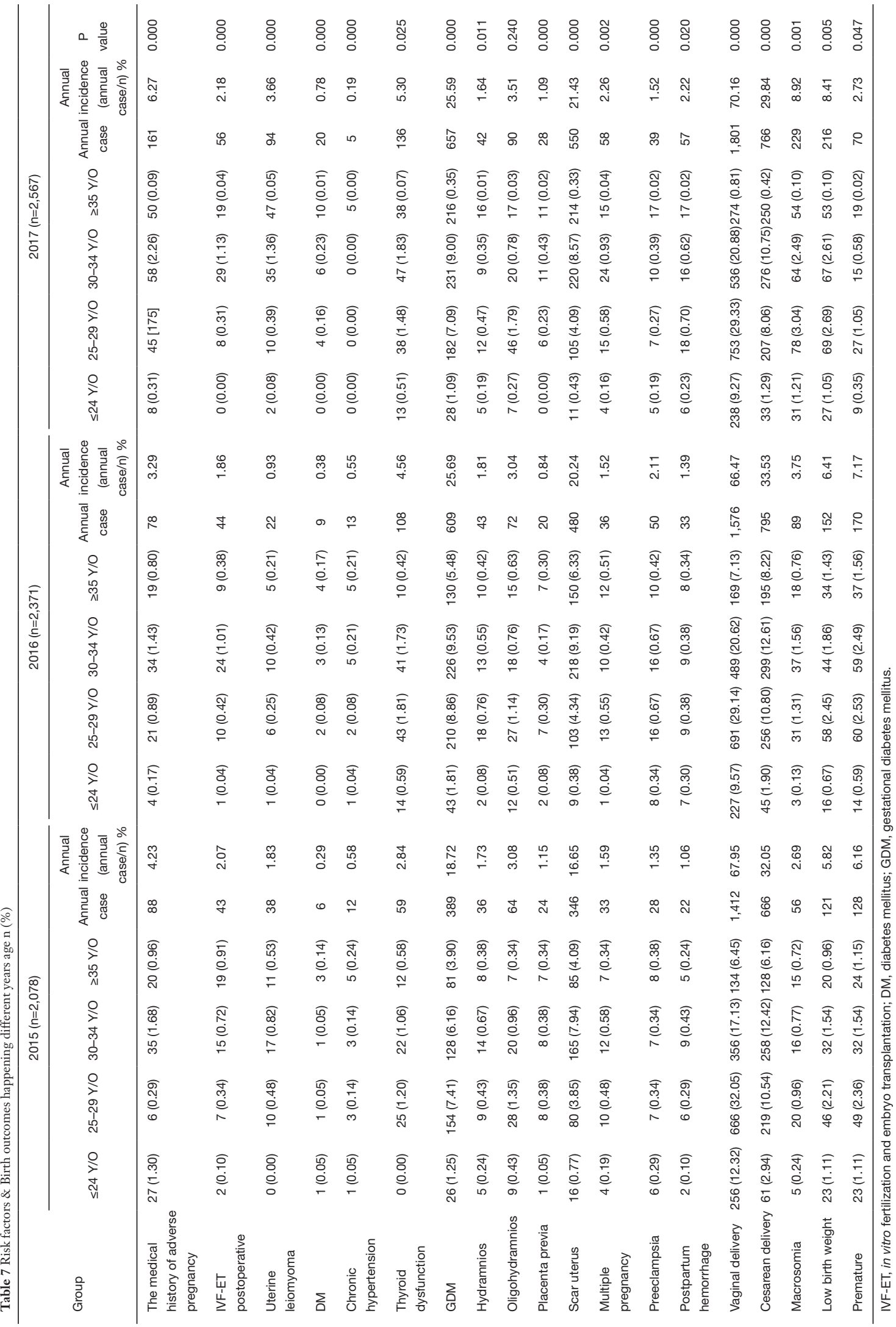


phenomenon was in consideration in 2016 women tried to conceive naturally, but because they had medical history of adverse pregnancy and chronic complications, they could not conceive as they wish, hence in order to reach the goal of reproduction, they had to turn to the ART. Thus, in 2017, the number of pregnancy by ART rose to the level in 2015 again.

With increased age, female incidence of reproductive system diseases, such as uterine leiomyoma, uterine adenomyosis and endometriosis etc. gradually increased. From the study, we found that maternal women over the age of 30 years old gradually increased the risk of uterine leiomyoma. The maternal incidence of pregnancy with uterine leiomyoma was $1.83 \%$ in 2015 ; fell to $0.93 \%$ in 2016, while significantly increased to $3.66 \%$ in 2017 . This may be due to the "comprehensive two-child" policy, which showed a growing number of advanced maternal age women involved in the case of pregnancy and birth. The maternal morbidity of uterine leiomyoma declined in 2016 because the women did not successfully get pregnant or simply have just had a baby conceived, so advanced maternal women found all the ways necessary to have a successful pregnancy and delivery, so in 2017, the rate increased accordingly.

Advanced maternal age also was a high risk factor for DM. Maternal pregnancy rate of diabetic was $0.29 \%$ in 2015 , then rose to $0.38 \%$ in 2016 , and 2017 rose slightly to $0.78 \%$, in the 3 years it slowly went up. This suggested that after the implementation of "two-child policy", the number of maternal diabetic pregnancies increased steady, considering its conception and smooth birth, which did not fluctuate among years.

Pregnancy with chronic hypertension is one of the pregnancy-induced hypertension diseases, belonging to the high-risk pregnancy. Epidemiological studies showed that with the growth of the age, the incidence of high blood pressure increased, and about $10 \%$ patients may have other diseases, like diabetes, chronic kidney disease, thyroid disease, and vascular disease. The incidence of chronic hypertension in advanced maternal age increased significantly. By the statistical results in this article, we saw that maternal pregnancy incidence of chronic hypertension was $0.58 \%$ in 2015 , and then slightly decreased from $0.55 \%$ in 2016 to $0.19 \%$ in 2017 , it was on the decline in the 3 years. It may because the contemporary women pay more attention to the examination and health care of pregnancy, with the growth of blood pressure monitoring concerns. They had already been interposed early in their pregnancy by controlling the blood pressure in the whole pregnancy.
Clinical thyroid dysfunction includes hyperthyroidism, hypothyroidism, subclinical thyroid dysfunction and other diseases. Without the control, thyroid dysfunction may lead to menstrual disorders, low conception rate, gestational hypertension disease, abortion, premature delivery, fetal growth restriction, etc., even the maternal thyroid function may have profound effects on the development of the babies' intelligence (16). As early as 2011, the Chinese medical association branch of obstetrics and gynecology maternity committee released before pregnancy and pregnancy care guide (version 1) (17), thyroid function has become a reference project during pregnancy, and our samples also had launched the project in the antenatal check. In this paper, the study found that older than 30 years old, the pregnant women rate of thyroid dysfunction increased. From the statistical results, the rate of pregnancy with abnormal thyroid function was $2.84 \%$ in 2015 , rose to $4.56 \%$ in 2016 , and up to $5.30 \%$ in 2017 , there was an upward trend in these 3 years. This may be due to more attention was paid to maternal pregnancy checks, and thyroid function check project popularized gradually, then the detection rate increased. It brought the positive clinical significance on the early diagnosis and early treatment for pregnancy with thyroid disease.

Gestational diabetes is a common obstetric complication, which has bad influences on many organs, such as heart, kidney, and brain for the pregnant woman, and it can significantly increase the risk of adverse pregnancy outcomes, even may endanger the maternal fetal lives. In this paper, the study showed that the increase of gestational age for the onset of GDM effect is obvious, the greater the age, the higher the incidence of gestational diabetes. The incidence of GDM was $18.72 \%$ in 2015 , rose to $25.69 \%$ in 2016 , and remained at $25.59 \%$ in 2017 , which basic flat in 2016 and 2017, but rose significantly than in 2015. This may be due to the two-child policy, more and more women, especially advanced women, joined the team to have two children.

Amniotic fluid is an important part in maintaining fetal life, hydramnios or oligohydramnios can both affect fetal or maternal health. From the statistical results in this article, the incidences of hydramnios in 3 years were basically unchanged. This may be due to the current antenatal examination paid attention to the monitoring of amniotic fluid, especially at high risk of maternal pregnancy supervision. In the process of antenatal examination, we should detect and treat abnormal amniotic fluid timely, and terminate pregnancy in a proper way, to ensure the safety of 
the mothers and infants.

In recent years, with the rising rate of the application of ART, artificial abortion operations and cesarean deliveries, the incidence of placenta previa also gradually increased. Placenta previa is a serious pregnancy induced complications and one of the common causes of bleeding in late pregnancy. By the statistical results it can be seen that the incidence of placenta previa was $1.15 \%$ in $2015,0.84 \%$ in 2016, and rebounded to $1.09 \%$ in 2017 , close to 2015 . The year 2016 was seemed to be a relatively "safe" year, this may because the year 2016 was the first year to be fully opened to the two-child policy. The advanced aged women who once had history of a miscarriage or caesarean tried to conceive. While in 2017, maternal women with high risks had a successful pregnancy and birth, maybe that is the reason of placenta previa had a rising situation.

Scar uterus is always left as a result of postoperative uterine procedures. From this statistical result, the incidence of scar uterus in 2015 was $16.65 \%$, significantly increased to $20.24 \%$ in 2016 , and continued to rise to $21.43 \%$ in 2017 , there was an upward situation in these 3 years. This may be due to changes in national childbearing policy; the women once had histories of uterine surgeries considered pregnancy again, resulting in the increase of the scar uterus.

Multiple pregnancy refers to that there are two or more fetuses in a pregnant uterine cavity at the same time, which can be attributed by age, genetic factors, and also may be caused by drugs. This study shows that in advanced pregnant women, a significant increase in the risk of multiple pregnancy, this may inseparable be the advanced women with reproductive purposes chose ART. Multiple pregnancies are considered to be one of the main risks factors associated with drugs that promote ovulation and ART. Maternal incidence of multiple pregnancies was $1.59 \%$ in 2015 , and 2016 remained at $1.52 \%$, rose to $2.26 \%$ in 2017, and that rose obviously in 2017 than the foregoing 2 years. The changes of incidence were closely related to the ART. Considering to policy, in 2016 the multiparas tried to conceive naturally, but because of all kinds of complications, they couldn't conceive, hence they had to resort to ART in order to reach their goal of reproductive, so the incidence of multiple pregnancies significantly climbed in 2017 .

Preeclampsia is an idiopathic disease during pregnancy, and the illness can rapidly develop, leading to severe viscera function damage or fetal complications. The result showed that the incidence of maternal preeclampsia is $1.35 \%$ in 2015 , rose to $2.11 \%$ in 2016 , and fell to $1.52 \%$ in 2017 . This may be due to the current obstetrics outpatient service to strengthen the supervision over the high risk of maternal pregnancy, timely screening high-risk groups. Postpartum hemorrhage is always in the leading cause of maternal deaths in the world. By this paper it can be seen that the rate of postpartum hemorrhage in advanced age was higher than other age groups, which may be due to the increasing proportion of advanced maternal women year by year, followed by the rising rate of postpartum hemorrhage.

In the statistical results, the rate of vaginal births was $67.95 \%$ in 2015 , slightly declined to $66.47 \%$ in 2016 , and then rose observably to $70.16 \%$ in 2017 ; corresponding to the cesarean delivery rate equal in 2015 and 2016, while fell to $29.84 \%$ in 2017 . Although under the push of the childbearing policy, increasing age, high risk of maternal complex cases, but cesarean rate in 2017 dropped instead. This may be due to the obstetricians pay more attention to the early intervention on the stage of labor, avoid unnecessary cesarean sections, and more strictly to control the indications of cesarean section, strengthen vaginal midwifery technique, to a certain extent, reduce the rate of cesarean delivery. Thus, it is strongly advised that primiparas choose vaginal delivery as much as possible (18).

The occurrence of macrosomia may not only increase the maternal and infant intrapartum complication, but may increase the adult suffering from asthma and allergic disease, cancer and other risks. The statistics shows that, the incidence of maternal childbirth macrosomia was increased year by year from 2015 (2.69\%) to 2017 (8.92\%). Due to the gradual opening of the "two-child" policy, the number of mothers is gradually increasing, and multiple pregnancies and multiple births are also one of the causes of the occurrence of macrosomia. In addition, the incidence of low birth weight maternal childbirth was also increased year by year from 2015 (5.82\%) to 2017 (8.41\%). From the study, we could see that the increasing proportion of advanced pregnant women, the gestational hypertension, abnormal thyroid function, abnormal placenta and assisted reproductive conception, which may be increased the incidence of low birth weight in among these 3 years (19).

Premature for the unsound immune function, immature organ development, lead to the poor quality of life, unknowable long-term prognosis, this is the main cause of morbidity and mortality in perinatal infants. From this paper, the incidence of maternal premature delivery was declined significantly from 2015 (6.16\%) to 2017 (2.73\%). This may be due to the unceasing progress of obstetric medical level which avoided the occurrence of premature birth. 
Above all, clinical workers should take inspection to health care work in the whole perinatal period, pay close attention to the change of vital signs, interpose the diagnosis and treatment for high-risk pregnant women timely, choose the best way of delivery duly, reduce the occurrence of neonatal anomalies, protect the health of maternal and child comprehensively according to different childbirth women.

\section{Conclusions}

The study indicated that after the childbearing policy change, maternal age ratio increased year by year. Between different years, different age groups, the incidences of various risk factors, the delivery modes and adverse pregnancy outcomes also changed. It may be due to the changes in childbearing policy, the age distribution proportion changed, which subsequently caused the changes in the incidence of risk factors. The advance of medical technology and socio-economics, also affected the changes of pregnancy risk factors, delivery modes and the pregnancy outcomes in different years.

\section{Acknowledgments}

Funding: Southern Medical University clinical research projects (No. LC2016PY035).

\section{Footnote}

Conflicts of Interest: All authors have completed the ICMJE uniform disclosure form (available at http://dx.doi. org/10.21037/apm.2020.04.27). The authors have no conflicts of interest to declare.

Ethical Statement: The authors are accountable for all aspects of the work in ensuring that questions related to the accuracy or integrity of any part of the work are appropriately investigated and resolved. The study was approved by ethics committee of The IRB of Zhujiang Hospital of Southern Medical University (No. 2018-FCK001), and all the participants have given informed consent before taking part.

Open Access Statement: This is an Open Access article distributed in accordance with the Creative Commons Attribution-NonCommercial-NoDerivs 4.0 International License (CC BY-NC-ND 4.0), which permits the noncommercial replication and distribution of the article with the strict proviso that no changes or edits are made and the original work is properly cited (including links to both the formal publication through the relevant DOI and the license). See: https://creativecommons.org/licenses/by-nc-nd/4.0/.

\section{References}

1. Zhang $X Q$, Huang $C$, Zhang $Q$, et al. Fertility intention for the second child under the selective and vniversal twochild policies: comparisons and implications. Popul Res 2016;40:87-97.

2. Buchanan A, Rotkirch A. Fertility rates and population decline: no time for children? London: Palgrave Macmillan, 2013:327.

3. Chen R, Gu BC. Transition of fertility desire in the past 30 years in Shanghai. Popul Society 2014;1:49-54.

4. Zhai ZW, Zhao MH. Causes and consequences of only the two child policy for only-child parents. Popul Family Planning 2014;3:10-2.

5. Zeng Y. Implementing the universal two-child policy as soon as possible is a win-win policy for both the nation and people. China Popul Today 2015;5:115-26.

6. Yao YM, Li F, Yin WY. Accumulated couples and fertility release under the new fertility policy. Popul Res 2014;7:3-18.

7. Luo C, Xu QH, Dai QY. The implementation of the new family planning policy and population development prospect in Yunnan Province. Chinese J Popul Sci 2014;3:30-42.

8. Yuan X. The changes of family planning policy and the balanced development of population in China. Popul Res 2016;38:5-14.

9. Shi ZL, Yang YY. Fertility intention and fertility behavior in Huber province under the new two-child policy. Popul Res 2014;5:27-40.

10. Feng XT. Desires and its factors for bearing the second child of urban couples who are both only child. J Social Sci 2010;5:59-66.

11. Zhuang $Y$, Jiang $Y$, Wang ZL. Fertility intention of rural and urban residents in China: results from the 2013 national fertility intention survey. Popul Res 2014;3:3-13.

12. Wei Y, Liu XL, Zhang C. Effect of maternal age on pregnancy a retrospective cohort study 1 . Chin Med J (Engl) 2014;127:2241-6.

13. Hurley EG, DeFranco EA. Influence of paternal age on perinatal outcomes. Am J Obstet Gynecol 2017;217:566.e1-6.

14. Cakmak Celik F, Aygun C, Kucukoduk S, et al. Maternal 
and neonatal outcomes in advanced maternal age: a retrospective cohort study. J Matern Fetal Neonatal Med 2017;30:2452-6.

15. Fuchs F, Monet B, Ducruet T, et al. Effect of maternal age on the risk of preterm birth: a large cohort study. PLoS One 2018;13:e0191002.

16. Zhao Y. Changes in risk factors of high risk pregnancy after the two child policy. China Med Pharm 2017;148:64-7.

17. Obstetrics Group of Gynecology and Obstetrics

Cite this article as: Teng $\mathrm{X}$, Shane MI, Pan S. The changing situation about maternal age, risk factors and pregnancy outcomes after the two-child policy: a retrospective cohort study. Ann Palliat Med 2020;9(3):824-834. doi: 10.21037/ apm.2020.04.27
Department in Chinese Medical Association. Health care guidelines for pregnancy and pregnancy (First Edition). Chinese J Obstetrics Gynecol 2011;46:150-3.

18. Deng SQ, Chen HT, Wang DY. Changes of cesarean section rate and caesarean section indication before and after implementation of two-child policy. Chinese J Perinatal Med 2017;20:47-50.

19. Aldous MB, Edmonson MB. Maternal age at first childbirth and risk of low birth weight and preterm delivery in Washington State. JAMA 1993;270:2574-7. 